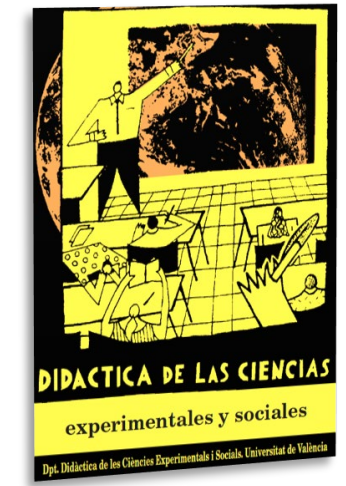

\title{
La construcción de la ciudadanía en la formación inicial del profesorado de Educación Infantil
}

\author{
The construction of citizenship in initial \\ teacher training of Early Childhood Education
}

DOI: $10.7203 / D C E S .39 .17121$

\author{
Francisco F. García Pérez \\ Universidad de Sevilla, ffgarcia@us.es \\ ORCID iD: http://orcid.org/0000-0002-9988-3231 \\ María Burgos Sánchez \\ ONG Madre Coraje (España), mburgos@madrecoraje.org
}

\author{
Alicia Guerrero Fernández \\ Universidad de Sevilla, aliciaguerrero@us.es
}

\begin{abstract}
RESUMEN: Los graves problemas sociales y ambientales de nuestro mundo exigen un modelo de educación para una ciudadanía global, participativa y crítica. Para ello es necesaria una formación del futuro profesorado como educadores en ciudadanía, desarrollando la concienciación en relación con esos problemas y las actitudes de compromiso social, con un modelo didáctico que propicie la construcción de dicho modelo de ciudadanía en su futuro alumnado. Sobre esta fundamentación, se desarrolla una investigación cualitativa, mediante entrevistas grupales, que analiza el proceso de construcción de la ciudadanía en estudiantes del grado de Maestro en Educación Infantil de la Universidad de Sevilla, en el marco de una asignatura de educación para la ciudadanía. Los resultados muestran una evolución de la concepción de ciudadanía en los estudiantes hacia un modelo más participativo y vinculado al compromiso social. Un factor importante de esa evolución ha sido el modelo didáctico aplicado por el docente universitario.
\end{abstract}

PAlabras ClAVE: educación para ciudadanía, formación del profesorado, Educación Infantil, función social de la universidad, problemas del mundo.

\begin{abstract}
The severe social and environmental problems in our world demand a model of education for a global, participative and critical citizenship. This requires training future teachers as educators in citizenship, developing awareness in relation to these problems and attitudes of social commitment, with a teaching model that favors the construction of this model of citizenship in their future students. On this basis, a qualitative research has been developed, through group interviews, which analyses the process of construction of citizenship in students of the degree of Early Childhood Education Teacher at the University of Seville, within the framework of a subject on education for citizenship. The results show an evolution of the conception of citizenship in students towards a more participative model linked to social commitment. An important factor in this evolution has been the teaching model applied by the university teacher.
\end{abstract}

KEYWORDS: citizenship education, teacher training, Early Childhood Education, social function of the university, global problems.

Fecha de recepción: abril de 2020

Fecha de aceptación: junio de 2020 


\section{MARCO TEÓRICO}

Hoy nuestro mundo está afectado por un conjunto de graves problemas de carácter económico, social, político y, especialmente, ambiental, de tal envergadura que podemos hablar de "crisis civilizatoria" y de "emergencia planetaria". A esta situación se ha llegado por la confluencia de múltiples factores, interrelacionados, entre los que se pueden destacar: un crecimiento demográfico acelerado (aunque irregular según zonas del mundo), un desarrollo tecnológico basado en una explotación incontrolada de los recursos; un crecimiento económico que se pretende ilimitado, en un planeta finito... En definitiva, una dinámica con un claro desajuste de las relaciones sociedadnaturaleza. Las consecuencias son bien conocidas, siendo las más graves y perceptibles el agotamiento de recursos fundamentales para la Humanidad, la pérdida masiva de la biodiversidad, la generación de ingentes cantidades de residuos y, especialmente, el calentamiento global, que está generando un cambio climático quizás imposible de revertir. Y todo ello con las inevitables secuelas de desigualdades y enfrentamientos sociales. Este escenario exige redefinir los modelos de ciudadanía y de educación para la ciudadanía, necesarios para afrontar la situación. Ello implica, asimismo, abordar con una nueva mirada la formación del profesorado que tendrá la responsabilidad de educar a la ciudadanía del futuro.

\subsection{Ciudadanía, educación y profesorado}

Constituye, en efecto, una auténtica urgencia formar a los habitantes del mundo en y para una ciudadanía global, que promueva una conciencia respecto a los problemas citados y una actitud de compromiso con la resolución de los mismos (García Pérez, Moreno Fernández y Rodríguez Marín, 2015; Tonge, Mycock, \& Jeffery, 2012). Frente a una emergencia global se necesita una ciudadanía global, y para lograrla es imprescindible promover una educación para ser habitantes del planeta en el siglo XXI, siendo conscientes de que los actos individuales tienen repercusión sobre los problemas globales.

La preocupación por la formación ciudadana de niños y jóvenes ha sido objeto de múltiples estudios y declaraciones por parte de las instituciones internacionales (Guérin, Ploeg, \& Sins, 2013; Schulz et al., 2017). Algunos hitos claves han sido las propuestas de la ONU en relación con los "Objetivos de Desarrollo del Milenio", proclamados en el año 2000 (véase el informe final: Naciones Unidas, 2015) y con la "Década de la Educación para el Desarrollo Sostenible (2005-2014)" (véase el informe final: UNESCO, 2014); o las iniciativas de la Unión Europea, como la declaración de 2005 como "Año Europeo de la Ciudadanía a través de la Educación" (véase información relacionada en Comisión Europea, 2005). Asimismo, la educación para la ciudadanía global ha sido asumida por la UNESCO como una línea pedagógica básica de sus actuaciones (Wintersteiner, Grobbauer, Diendorfer, \& Reitmair-Juárez, 2015).

Más allá de las declaraciones oficiales, asumir este concepto de ciudadanía supone un profundo cuestionamiento de lo que se suele entender por "ciudadanía activa" o por "ciudadanía democrática", etiquetas bajo cuyo amparo, paradójicamente, la ciudadanía puede llegar a quedar desprovista de sus mejores potencialidades, como las relacionadas con la participación ciudadana y el compromiso con los problemas sociales (Bolívar, 2016; Romero Morante, 2018). Una auténtica ciudadanía democrática tiene que ser entendida como un proceso en continua construcción. Como expresa J. Romero Morante: "La democracia no es un destino alcanzado, sino una forma de caminar con los demás". Por tanto, "una ciudadanía activa y participativa no puede sino vivir en un estado de educación política continuada. [...] En definitiva, la educación de la ciudadanía es inherente a, y una condición sine qua non de, una democracia digna de ese nombre" (2012, p. 258).

En la construcción de la ciudadanía son, pues, mucho más efectivas las experiencias vividas que la mera transmisión de conocimientos. Lo recogen algunas investigaciones sobre la infancia (Novella, Agud, Llena y Trilla, 2013) y también sobre jóvenes y adultos (Daly, Schugurensky, \& 
Lopes, 2009; Tonge, Mycock, \& Jeffery, 2012). Esa educación vinculada a la experiencia participativa se desarrolla en contextos sociales diversos y en todos los tramos de edad. Un reto tan exigente no puede ser responsabilidad única del sistema educativo, pero sin duda su papel es fundamental. Por consiguiente, la educación para una ciudadanía activa y participativa debería ser asumida en la formación escolar ${ }^{1}$, integrándola, de forma transversal, en las diversas materias e incorporando otras aportaciones de la educación no formal (García Pérez, 2018).

La asunción de esta perspectiva no es fácil de llevar a la práctica, pues - como se ha dicho- no es una cuestión de transmisión sino de participación real, lo que implica actitudes y acciones orientadas por valores. Y ello resulta difícilmente compatible con las características de la estructura escolar, un contexto que tiende a desvincularse de las realidades sociales y que está preparado, más bien, para manejar saberes tradicionalmente establecidos (García Pérez, 2018). A las limitaciones propias de la cultura escolar dominante se une la vigencia de una cultura docente tradicional, que con frecuencia privilegia el rol de experto disciplinar sobre la dimensión de educador. Ello se manifiesta en declaraciones y comportamientos del profesorado, como muestran diversas investigaciones (De Alba Fernández y García Pérez, 2017; García Pérez, 2018).

Pero no se puede desarrollar una educación para la ciudadanía como la que se postula si no hay profesionales concienciados y preparados para ello. Hoy no es suficiente con un docente que se limite a ofrecer informaciones de carácter cívico, pues afrontar los graves problemas del mundo implica el desarrollo de actitudes y valores, que no se transmiten como un contenido neutral sino ofreciendo modelos de referencia. En ese sentido, la participación cívica y política constituye un bagaje personal fundamental para el profesorado que ha de enseñar ciudadanía (Schugurensky \& Myers, 2003). De ahí la necesidad de que los futuros educadores y educadoras se formen en esa conciencia y esa responsabilidad, mostrándose como modelos en los que sus alumnos puedan verse reflejados (García Pérez, 2018).

Así, como marco de referencia general, proponemos el modelo de formación docente del Proyecto IRES ${ }^{2}$, un modelo basado en el tratamiento de "problemas prácticos profesionales", es decir, los problemas básicos a los que se enfrentan los docentes en el ejercicio de su profesión, en especial el diseño y desarrollo de propuestas didácticas de educación ciudadana enfocadas como "tratamiento de problemas" (García Pérez, 2006). De esta forma, los futuros docentes se preparan mejor para educar a los ciudadanos en saber afrontar esos problemas de manera crítica y participativa. En ese sentido, entendemos que la formación ciudadana y la formación docente responden a unas mismas necesidades y comparten también metas y modelos (García Pérez, 2018).

\subsection{Universidad y formación docente}

La universidad, como etapa superior de la educación reglada, tiene una especial responsabilidad en la formación de ciudadanas y ciudadanos conscientes y comprometidos socialmente, desde sus futuras profesiones, en la construcción de una ciudadanía global. Ese papel de la universidad ha sido destacado desde instancias diversas. Lo había declarado la primera Conferencia de la UNESCO sobre Educación Superior en el siglo XXI (UNESCO, 1998) y se ratificó en una segunda Conferencia (UNESCO, 2009). Por su parte, en la universidad española, también se ha destacado el contexto universitario como un espacio privilegiado para formar de manera integral a las personas, por lo que la propia institución tendría que reformular sus estrategias de docencia, aprendizaje, investigación y

\footnotetext{
${ }^{1}$ No nos referimos a la incorporación al currículum de una asignatura determinada -que ya de por sí ha sido un proceso polémico en España (Gómez-Rodríguez y García-Ruiz, 2019)- sino a una opción más profunda y transversal.

${ }^{2}$ El Proyecto IRES (Investigación y Renovación Escolar) es un programa de investigación y acción educativas, vigente desde 1991. El IRES sirve, asimismo, como marco para el desarrollo profesional del profesorado. Para conocimiento del proyecto, puede consultarse García Pérez, 2000 y García Pérez y Porlán, 2000. Asimismo, puede visitarse la web de la Red IRES: www.redires.net
} 
transferencia, además de su función social (Observatorio de la Cooperación Universitaria al Desarrollo, 2016).

En ese sentido, la Responsabilidad Social Universitaria (RSU) aparece como una idea clave para el logro de tales fines (Martínez-Usarralde, Lloret-Catalá y Mas-Gil, 2017). La RSU se enmarca en la idea más general de "Responsabilidad Social", como paradigma global para interpretar los nuevos fenómenos mundiales. La UNESCO (2009) destaca la necesidad de un liderazgo social de la universidad para abordar los nuevos problemas, como el cambio climático, la gestión del agua, el desafío intercultural, etc. Ello supone una apelación a la ciudadanía global desde una perspectiva de contribución a la paz, a la defensa de valores éticos y democráticos y de derechos humanos (MartínezUsarralde, Lloret-Catalá y Mas-Gil, 2017).

Mediante la "Estrategia Universidad 2015" el Ministerio de Educación español considera la RSU como un eje de la actuación de la universidad hacia la meta de un Desarrollo Humano Sostenible. Ello requiere "un nuevo modelo educativo acorde a las expectativas sociales y basado en los valores de sostenibilidad, igualdad, solidaridad, entre otros". Asimismo, exige "nuevas metodologías y formación previa del profesorado en el desarrollo de las competencias para la capacitación socialmente responsable del alumnado" (Martínez-Usarralde, Lloret-Catalá y Mas-Gil, 2017, p. 15).

En este marco la educación de ciudadanos críticos constituye un objetivo central de la formación universitaria (De Manuel Jerez y Donadei, 2018). Por tanto, lo lógico -frente a lo que realmente ocurre- es que los contenidos de carácter transversal, como los relativos a la educación para la ciudadanía, estén presentes en los planes de estudio de las carreras universitarias. Propugnamos, pues, un modelo de "universidad ciudadana", una universidad realmente comprometida con la sociedad, que conjugue de manera compleja las funciones universitarias de educar, investigar y transformar (Manzano-Arrondo, 2011).

Si la formación de ciudadanos críticos y de profesionales comprometidos es una responsabilidad general de la universidad para con la sociedad, mucho más lo es en el caso específico de la formación de los futuros docentes. Para desarrollar una educación para la ciudadanía como la caracterizada anteriormente es indispensable que haya profesoras y profesores capacitados para hacerlo. Y en ese sentido su formación inicial es una fase importante. Sin embargo, en general, la formación universitaria no prepara al futuro profesorado para ello. De hecho, los resultados de investigación muestran diversos déficits en ese aspecto (Álvarez-García, Sureda-Negre y ComasForgas, 2018; Bourn, Huntan, \& Bamber, 2017; Estellés y Romero, 2018; Molina, Miralles y Ortuño, 2013).

Por consiguiente, la universidad debería intentar que la formación que reciben los futuros docentes les ayude a ser auténticos educadores de ciudadanos críticos y participativos, que trabajen por la justicia social (González Valencia y Sant, 2014; Guérin, Ploeg, \& Sins, 2013; Krichesky, Martínez-Garrido, Martínez Peiret, García Barrera, Castro Zapata y González Bustamante, 2011). Ello debería ser responsabilidad del currículum formativo en su conjunto; en todo caso, también es conveniente que haya materias que aporten una formación específica. Es el caso de la asignatura en la que se ha desarrollado esta investigación, "Ciudad y Ciudadanía en la Educación Infantil".

Así, pues, en coherencia con lo que se ha expuesto, la formación de los futuros docentes debería desarrollarse trabajando sobre propuestas de enseñanza que sigan un modelo didáctico investigativo (García Pérez y Porlán, 2017), basado en el tratamiento de problemas sociales y ambientales relevantes para la ciudadanía (Pagès y Santisteban, 2011). Y ello se realizaría en el marco general antes citado- de trabajo sobre "problemas prácticos profesionales" (García Pérez, 2006). La validez de este modelo formativo viene confirmada por los resultados de diversas investigaciones, en las que se constata la potencialidad de los procesos de formación basados en el diseño y desarrollo de proyectos de educación para la participación ciudadana, y apoyados en actividades de reflexión y en el acompañamiento colaborativo de formadores (Estepa, 2012; García Pérez, De Alba Fernández y Navarro Medina, 2015; Willemse, Ten Dam, Geijsel, Van Wessum, \& Volman, 2015). 
En definitiva, consideramos que la formación ciudadana y la formación docente son indesligables y deberían desarrollarse siguiendo un modelo de tratamiento y resolución de problemas. Ese es el marco de referencia que ha orientado la investigación empírica que a continuación se presenta.

\section{Metodología}

\subsection{Contexto, problemas de investigación y metodología aplicada}

La investigación que se expone se basa en el seguimiento del desarrollo de la docencia de la asignatura "Ciudad y ciudadanía en la Educación Infantil", a través de las declaraciones del alumnado implicado, en los cursos 2014-2015 y 2015-2016. Se trata de una asignatura optativa cuatrimestral (de 6 créditos ECTS: 4 teóricos / 2 prácticos) que se imparte en el primer cuatrimestre del curso $3^{\circ}$ del grado de Maestro de Educación Infantil, en la Facultad de Ciencias de la Educación de la Universidad de Sevilla.

La asignatura se desarrolla siguiendo el mismo modelo de metodología que se pretende sea aprendido por el alumnado (futuro profesorado), un modelo de orientación constructivista basado en el tratamiento de problemas sociales y ambientales (García Pérez, 2006; García Pérez y Porlán, 2017). En cada tema o unidad didáctica se parte del planteamiento de un problema, estimulando la explicitación de ideas de los estudiantes en relación con la temática, se trabaja, a continuación, sobre la documentación disponible (compendio de documentos, artículos y material audiovisual), mediante actividades de exposición, debates y elaboración progresiva de conclusiones, para llegar finalmente a aportar respuestas al problema planteado, que se recogen en sucesivos informes de conclusiones. En las actividades se combina el trabajo en pequeños grupos (como estrategia básica) con el trabajo en gran grupo y el trabajo individual.

Este modelo metodológico básico se aplica en las cuatro unidades didácticas de la asignatura, mediante una programación de actividades que conforman un proyecto de trabajo con la siguiente lógica. Se parte del problema base en la asignatura: ¿Qué sentido tiene "educar para ser ciudadanos/as" de nuestro mundo a los niños y niñas de Educación Infantil?; ello permite conocer los graves problemas sociales y ambientales del mundo y proponer un modelo de educación para una ciudadanía global desde la infancia. Dado que los futuros y futuras docentes tendrán que trabajar teniendo como referencia el currículum oficial y, probablemente, con los materiales didácticos más usuales, una segunda unidad se dedica al análisis del currículum oficial y de materiales didácticos convencionales, valorando críticamente las dificultades y las posibilidades trabajo. El siguiente paso consiste en conocer y analizar proyectos y experiencias innovadoras de educación para la ciudadanía (La ciudad de los niños, de F. Tonucci, el programa Ecoescuelas, Comunidades de aprendizaje, Filosofía para niños, etc.). El conocimiento de estas propuestas innovadoras, junto con las aportaciones en vivo de algunas maestras de Infantil, constituyen la base para la fase última del programa: la elaboración por los grupos de trabajo de un proyecto o diseño didáctico de educación para la ciudadanía para la etapa de Educación Infantil. Estos diseños son expuestos y compartidos por toda la clase y, cuando hay posibilidades, son aplicados en la fase de prácticas en los centros escolares.

El problema central de la investigación, que se corresponde con el objetivo básico de la misma, es el siguiente: ¿Cómo es el proceso de construcción de la ciudadanía global en el alumnado universitario del grado de Maestro de Educación Infantil? Dicho problema se aborda en el marco de un modelo de formación del profesorado estructurado en torno al tratamiento de "problemas prácticos profesionales" (García Pérez, 2006), considerando, en este caso, la construcción del concepto de ciudadanía y del modelo de educación para la ciudadanía como problemas que un (futuro) docente no puede dejar de plantearse. Este problema central se desglosa en tres preguntas de investigación 
más concretas: ¿qué capacidades debe tener y desarrollar un buen ciudadano (futuro docente) desde una perspectiva de ciudadanía global?; ¿cómo es el proceso de adquisición de dichas capacidades?; ¿qué aporta la formación universitaria -a través de la asignatura citada- a tal proceso de adquisición?

Para llevar a cabo este estudio se ha utilizado una metodología de carácter cualitativo (Bisquerra, 2009; Buendía, Colás y Hernández, 2012), que se ha considerado adecuada para el análisis de los fenómenos y procesos formativos citados. Más concretamente, el tratamiento de los datos se ha llevado a cabo mediante la técnica de análisis del contenido (Bardin, 1991; Krippendorff, 1990).

\subsection{Participantes e instrumentos}

La fuente básica de información para la investigación han sido los grupos de alumnos y alumnas de la clase objeto de estudio durante los dos cursos académicos citados. Para la recogida de informaciones del alumnado se ha utilizado la entrevista grupal (Buendía, Colás y Hernández, 2012), como instrumento coherente con la dinámica habitual de trabajo de grupos en la asignatura.

Las entrevistas grupales se realizaron, al inicio y al final de la docencia de la asignatura ${ }^{3}$, a dos grupos (de cuatro estudiantes cada uno) seleccionados como representativos de cada curso académico investigado. El aula del curso 2014-15 estaba constituida por 36 alumnas y alumnos, divididos en 12 grupos de trabajo; el aula de 2015-16 tuvo 64 estudiantes, divididos en 15 grupos. Más del $90 \%$ del estudiantado de estas aulas - como ocurre en el conjunto del grado de Maestro de Educación Infantileran mujeres, por lo que, de hecho, las participantes de todos los grupos entrevistados (numerados como 1, 2, 3 y 4) han sido de género femenino, siendo esta una variable que -por las características de la investigación- no hemos considerado como relevante a la hora de interpretar los resultados. Dado que la docencia se desarrolló según el mismo modelo en ambos cursos y que los grupos entrevistados reunían características similares, hemos considerado el conjunto de los cuatro grupos entrevistados como un conjunto único de participantes.

La entrevista grupal fue de carácter semiestructurado y el guion de la misma se elaboró teniendo en cuenta las temáticas básicas de la investigación, que sirvieron para establecer las categorías de análisis. Las cuestiones de la entrevista fueron sometidas a valoración de expertos y reajustadas en su formulación siguiendo las sugerencias de los mismos.

\subsection{Tratamiento de la información y sistema de categorías}

El tratamiento de la información procedente de las entrevistas grupales se ha realizado con la ayuda del programa Atlas.ti 8, empleando técnicas de análisis de contenido. Para la codificación en unidades de información, extraídas de las transcripciones de las entrevistas, se ha tenido como referencia un sistema de categorías diseñado a partir de los problemas investigados y teniendo en cuenta los referentes del marco teórico, sistema que se ha reajustado incorporando los aspectos emergentes durante el proceso de codificación. Este sistema de categoría (Tabla 1) se estructura en dos grandes macrocategorías, "Concepción de ciudadanía" y "Aprendizaje y evolución de la concepción de ciudadanía”, que, a su vez, incluyen otras categorías concretas. Para categorizar de forma más precisa las diferentes unidades de información, se han establecido, con una lógica de progresión, tres valores, que representan diferentes niveles de complejidad en cada una de las categorías.

\footnotetext{
${ }^{3}$ Las entrevistas iniciales se realizaron en octubre (tras las primeras clases, de inicio y contextualización, de la asignatura); las entrevistas finales se realizaron en junio, con la intención de que hubieran transcurrido varios meses tras la finalización de la asignatura (a finales de enero), para poder valorar mejor la consolidación de los aprendizajes básicos.
} 
TABLA 1. Sistema de categorías

\begin{tabular}{|c|c|}
\hline \multicolumn{2}{|c|}{$\begin{array}{l}\text { Macrocategoría "Concepción de ciudadanía" } \\
\text { Incluye las categorías } \mathrm{C}, \mathrm{R} \text { y } \mathrm{P} \text { (y sus respectivos valores). }\end{array}$} \\
\hline \multirow{3}{*}{$\begin{array}{l}\text { Categoría C: } \\
\text { Aproximación global al } \\
\text { concepto de ciudadano }\end{array}$} & $\begin{array}{l}\text { Valor } 1 . \text { Hace alusión principalmente a los derechos } \\
\text { vinculados al reconocimiento legal, a las normas o a } \\
\text { comportamientos cívicos convencionales. }\end{array}$ \\
\hline & $\begin{array}{l}\text { Valor } 2 \text {. Hace referencia también a los derechos y deberes, si } \\
\text { bien apreciándose cierta dimensión social y cultural de la } \\
\text { ciudadanía. }\end{array}$ \\
\hline & $\begin{array}{l}\text { Valor 3. Entiende el ejercicio de la ciudadanía como un } \\
\text { compromiso con la sociedad, identificando la interrelación de } \\
\text { los propios actos con las problemáticas globales. }\end{array}$ \\
\hline \multirow{3}{*}{$\begin{array}{l}\text { Categoría R: Relación } \\
\text { entre los propios actos y } \\
\text { hábitos individuales y las } \\
\text { problemáticas globales }\end{array}$} & $\begin{array}{l}\text { Valor 1. No se aprecia esta relación o bien se citan acciones } \\
\text { cívicas individuales aisladas. }\end{array}$ \\
\hline & $\begin{array}{l}\text { Valor } 2 \text {. Se reconoce que los propios actos pueden afectar a } \\
\text { otras personas o al entorno, pero se atribuye a factores } \\
\text { externos o al contexto la responsabilidad de cambiar la } \\
\text { situación. }\end{array}$ \\
\hline & $\begin{array}{l}\text { Valor 3. Identifica el valor y el poder de los actos cotidianos } \\
\text { en la transformación social, percibiéndose que se asume la } \\
\text { propia responsabilidad. }\end{array}$ \\
\hline \multirow{3}{*}{$\begin{array}{l}\text { Categoría P: Experiencias } \\
\text { de participación y } \\
\text { compromiso }\end{array}$} & $\begin{array}{l}\text { Valor 1. No ha participado, ha participado muy poco o resalta } \\
\text { las dificultades para participar. }\end{array}$ \\
\hline & $\begin{array}{l}\text { Valor 2. Ha participado periódicamente en iniciativas } \\
\text { sociales de atención a necesidades básicas con colectivos de } \\
\text { su entorno más cercano. }\end{array}$ \\
\hline & $\begin{array}{l}\text { Valor 3. Ha liderado o tomado iniciativas en pro de la justicia } \\
\text { social, estableciendo interrelaciones entre las actuaciones } \\
\text { locales y las consecuencias globales. }\end{array}$ \\
\hline \multicolumn{2}{|c|}{$\begin{array}{l}\text { Macrocategoría "Aprendizaje y evolución de la concepción de ciudadanía” } \\
\text { Incluye las categorías E y A (y sus respectivos valores). }\end{array}$} \\
\hline \multirow{3}{*}{$\begin{array}{l}\text { Categoría } \mathrm{E} \text { : Importancia } \\
\text { de la educación formal en } \\
\text { la construcción del propio } \\
\text { modelo de ciudadanía }\end{array}$} & $\begin{array}{l}\text { Valor 1. Atribuye esta función principalmente al ámbito } \\
\text { familiar y personal. }\end{array}$ \\
\hline & $\begin{array}{l}\text { Valor } 2 \text {. Valora de forma genérica la aportación de la } \\
\text { educación. }\end{array}$ \\
\hline & $\begin{array}{l}\text { Valor 3. Considera intrínseco al proceso educativo el } \\
\text { desarrollo de capacidades para el ejercicio de la ciudadanía, } \\
\text { sin minusvalorar el entorno familiar. }\end{array}$ \\
\hline \multirow{3}{*}{$\begin{array}{l}\text { Categoría A: Aportación de } \\
\text { la asignatura "Ciudad y } \\
\text { ciudadanía en la Educación } \\
\text { Infantil" a la construcción } \\
\text { del modelo de ciudadanía }\end{array}$} & $\begin{array}{l}\text { Valor } 1 \text {. No percibe la aportación de la asignatura a su } \\
\text { modelo de ciudadanía. }\end{array}$ \\
\hline & $\begin{array}{l}\text { Valor 2. Reconoce aportaciones puntuales de la asignatura a } \\
\text { su modelo de ciudadanía a través de ciertas actividades o de } \\
\text { comportamientos del docente. }\end{array}$ \\
\hline & $\begin{array}{l}\text { Valor 3. Reconoce que el modelo didáctico utilizado en la } \\
\text { asignatura le ha aportado un modelo global de ciudadanía. }\end{array}$ \\
\hline
\end{tabular}

Fuente: Elaboración propia

La interpretación de los resultados obtenidos del proceso de tratamiento mediante el sistema de categorías se realiza teniendo en cuenta la programación de la asignatura, antes esbozada. 


\section{RESUltados}

\subsection{Macrocategoría "Concepción de ciudadanía"}

Esta macrocategoría engloba las concepciones que tienen los y las estudiantes de Magisterio de Educación Infantil respecto a la ciudadanía, incluyendo tanto la perspectiva y el modelo de ciudadanía que han construido como el ejercicio y las experiencias que llevan a cabo en el marco de dicho modelo. Integra -como se indicó- las categorías C, R y P (y sus respectivos valores).

\section{Categoría C: Aproximación global al concepto de ciudadano}

Las unidades de información recogidas muestran la evolución en el nivel de formulación de las concepciones que el alumnado tiene respecto a la ciudadanía, así como los cambios desde las entrevistas iniciales a las finales.

GRÁFICO 1. Categoría C: Aproximación global al concepto de ciudadano

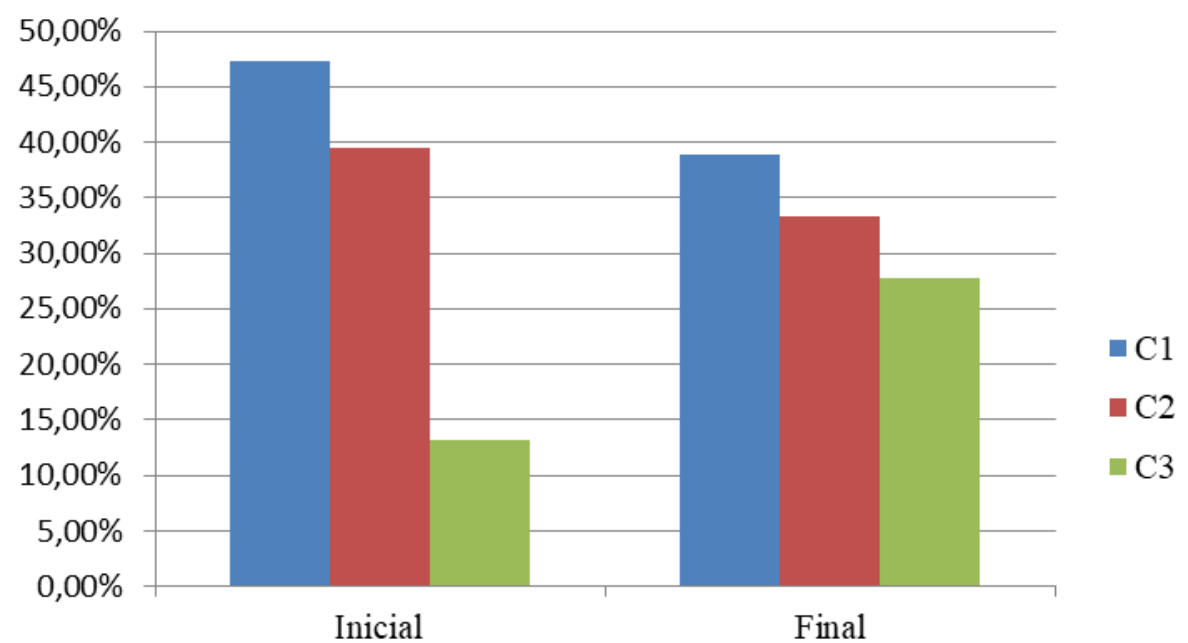

Fuente: Elaboración propia

Tal como queda reflejado en el gráfico 1, el nivel 1 (valor 1: nociones de ciudadanía que hacen referencia a derechos, normas y deberes, representado como C1) muestra un $47 \%$ de frecuencia en la entrevista inicial con respecto al 39\% de la final. Estos datos indican que se produce una evolución hacia formulaciones más complejas que se manifiestan en un segundo nivel (valor 2: C2), donde aparece una dimensión más sociocultural (39\% en la inicial y $33 \%$ en la final), alcanzándose un $28 \%$ de citas de nivel 3 (valor 3: C3) en la entrevista grupal final con respecto al 13\% que aparecía en la inicial; son concepciones donde se manifiesta ya un cierto grado de compromiso social e interrelación con las problemáticas globales.

Como ejemplos de unidades de información:

Valor 1: “(T): Comportarnos bien con los demás, cuidar todo lo que hay a nuestro alrededor, respetar. (R): Y respetar los derechos y cumplirlos.” [Alumnas T y R; Grupo 4, entrevista inicial].

Valor 3: "Pues para mí, ser ciudadano es ser una persona comprometida y participativa con los problemas que hay actualmente en la sociedad. Y ya no sólo eso, como ha dicho P, no sólo los problemas que le rodean, sino ahora en una sociedad que está tan globalizada, pues los problemas 
que hay en el mundo. No sólo los que nos afectan directamente sino también los que nos afectan indirectamente" [Alumna A; Grupo 1, entrevista final].

Categoría R: Relación entre los propios actos y hábitos individuales y las problemáticas globales

En esta categoría se aprecia la evolución entre las entrevistas iniciales y finales con respecto a la relación que establecen las estudiantes entre sus actos individuales y el impacto en los problemas globales. Tal y como puede verse en el gráfico 2, las respuestas de nivel 1 (donde las estudiantes no aprecian ninguna relación entre las acciones individuales y el impacto global, o donde dichas acciones se dan de manera aislada) resultan escasas en las entrevistas iniciales (5\%) y desaparecen en las finales $(0 \%)$, en favor de niveles o valores más elevados. El gráfico muestra que perciben que sus propios actos pueden afectar a su entorno, aunque mayoritariamente atribuyen a factores externos la responsabilidad de cambiar la situación, como se ve en las unidades de nivel 2, que suponen un $60 \%$ de las unidades de información en las entrevistas iniciales y un 76\% en las finales. Asimismo, las respuestas que se encuentran en un nivel avanzado (valor 3) muestran la incidencia de las acciones individuales en la transformación social, asumiendo de manera consecuente la propia responsabilidad; estas unidades tienen una importante presencia tanto en las entrevistas iniciales $(35 \%)$ como en las finales $(24 \%)$.

GRÁFICO 2. Categoría R: Relación entre los propios actos y hábitos individuales y las problemáticas globales

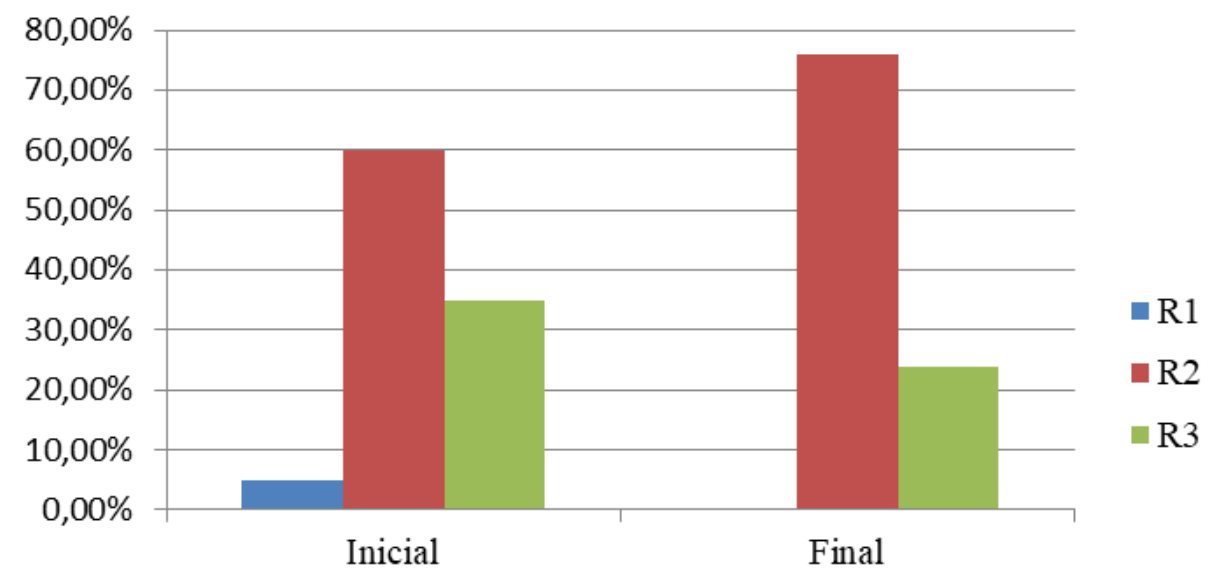

Fuente: Elaboración propia

Como ejemplos podemos ver:

Valor 2: "Es muy difícil porque yo creo que eso tiene que venir de la gente de arriba. Es decir, nosotros podemos poner nuestro granito de arena como personas a nivel individual o a nivel colectivo en un apartado de la sociedad, pero si después llega la gente de arriba, la más importante, y empieza a recortar en sanidad, en educación, en niños con discapacidad [sic]... entonces ahí estamos empezando a fallar a la ciudadanía, porque es que los grupos sociales están totalmente coartados dependiendo del grupo al que pertenezcas" [Alumna C2; Grupo 2, entrevista inicial].

Valor 3: "[...] yo creo que al final, de una manera o de otra, todos hemos hecho, hemos contribuido a que esto esté sucediendo, de manera más directa o indirecta, pero algo siempre... Yo pienso que la culpa no se la debemos dar solamente a una persona. La culpa es de todos porque esto es una sociedad y todos somos ciudadanos, y si está pasando algo es porque todos no hemos remado hacia un mismo sitio. Aquí estamos, cada uno mirando por nuestros propios 
intereses y, al final, lo que estamos consiguiendo es que se destruya todo lo que anteriormente han ido construyendo para que tengamos un buen fututo, y nosotros mismos nos lo estamos cargando" [Alumna S; Grupo 3, entrevista inicial].

\section{Categoría P: Experiencias de participación y compromiso}

En el gráfico 3 se muestran las frecuencias de respuestas referidas a las vivencias y experiencias de participación que el alumnado ha tenido. El primer nivel, que representa la casi nula participación y/o la mención de los obstáculos que la han impedido, aparece con un $72 \%$ en las entrevistas iniciales $\mathrm{y}$, sorprendentemente, con un $79 \%$ en las finales, donde las participantes reflejan un bajo grado de implicación. Con respecto al nivel 2 (donde se mencionan experiencias participativas periódicas en iniciativas sociales del entorno más cercano), las unidades de información de las entrevistas iniciales (28\%) disminuyen a la mitad en las finales (14\%). No hay respuestas de nivel 3 en las entrevistas iniciales y también son escasas (7\%) en las finales; este nivel 3 supone un grado de participación más avanzado donde se pueden dar experiencias de liderazgo y de toma de iniciativas basadas en la justicia social y donde se reconoce la repercusión de las intervenciones locales en las consecuencias globales.

GRÁFICO 3. Categoría P: Experiencias de participación y compromiso

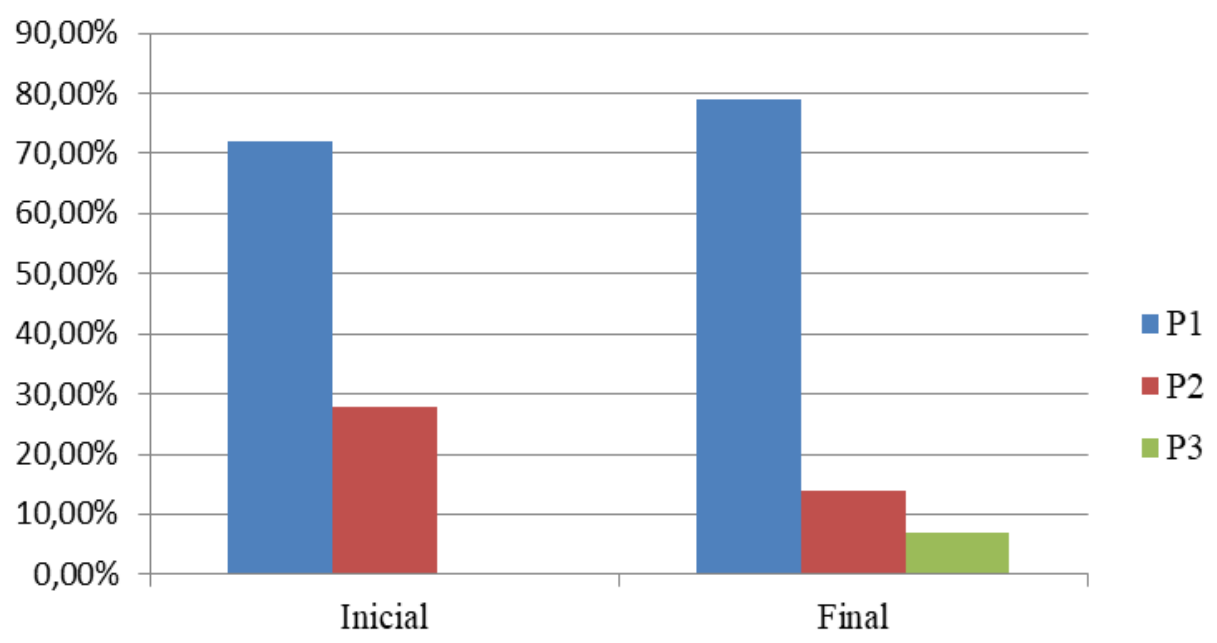

Fuente: Elaboración propia

Algunos ejemplos:

Valor 1: "Hombre, yo la verdad es que no participo mucho y esas cosas, no voy ni a votar ni... [...] No participo ni... vivo y ya está (risas). Esas veces yo digo: 'es que yo quiero participar y es que no me dejan', nada más que me dejan votar y elegir a quien yo quiero que esté, que al final puede salir o no salir o no gustarme el que sale y al final esa persona no me está dejando a mí participar en lo que yo quiero. Y luego ya, si es voluntariado y esas cosas así, siempre quiero hacer, pero siempre me entero cuando ya se ha acabado el plazo de inscripción o algo y siempre me entero cuando alguien me dice: 'ay, voy a hacer un voluntariado de no sé qué...'. Y yo siempre digo: 'o yo miro mal o es que nunca me entero de nada'..." [Alumna B; Grupo 1 , entrevista inicial].

Valor 3: "[...] y luego desde mi vida profesional, futura vida profesional, pues también he llevado a cabo un proyecto con los niños sobre la educación para el desarrollo. Entonces he intentado participar y más que nada sensibilizar a los niños con temas que yo creo que todo ciudadano debe tener. Con valores sobre el medio ambiente, sobre el consumo responsable, la pobreza y no sé, yo de esa forma creo que yo he participado en esos problemas y creo que 
también yo he hecho a los niños partícipes de los problemas actuales" [Alumna A; Grupo 1, entrevista final].

\subsection{Macrocategoría "Aprendizaje y evolución de la concepción de ciudadanía"}

Esta segunda macrocategoría hace referencia a las fuentes de la construcción de la ciudadanía y al proceso de enseñanza y aprendizaje de dicha ciudadanía en los individuos, así como al papel de la educación formal, y, en este caso, la universitaria (más concretamente la asignatura de "Ciudad y ciudadanía en la Educación Infantil"), en relación con el aprendizaje del modelo de ciudadanía. Integra las categorías $\mathrm{E}$ y A (y sus respectivos valores).

Categoría E: Importancia de la educación formal en la construcción del propio modelo de ciudadanía

La categoría analizada en gráfico 4 hace referencia al grado de relevancia que el alumnado otorga al papel de la educación formal en su formación como ciudadanos y ciudadanas.

GRÁFICO 4. Categoría E: Importancia de la educación formal en la construcción del propio modelo de ciudadanía

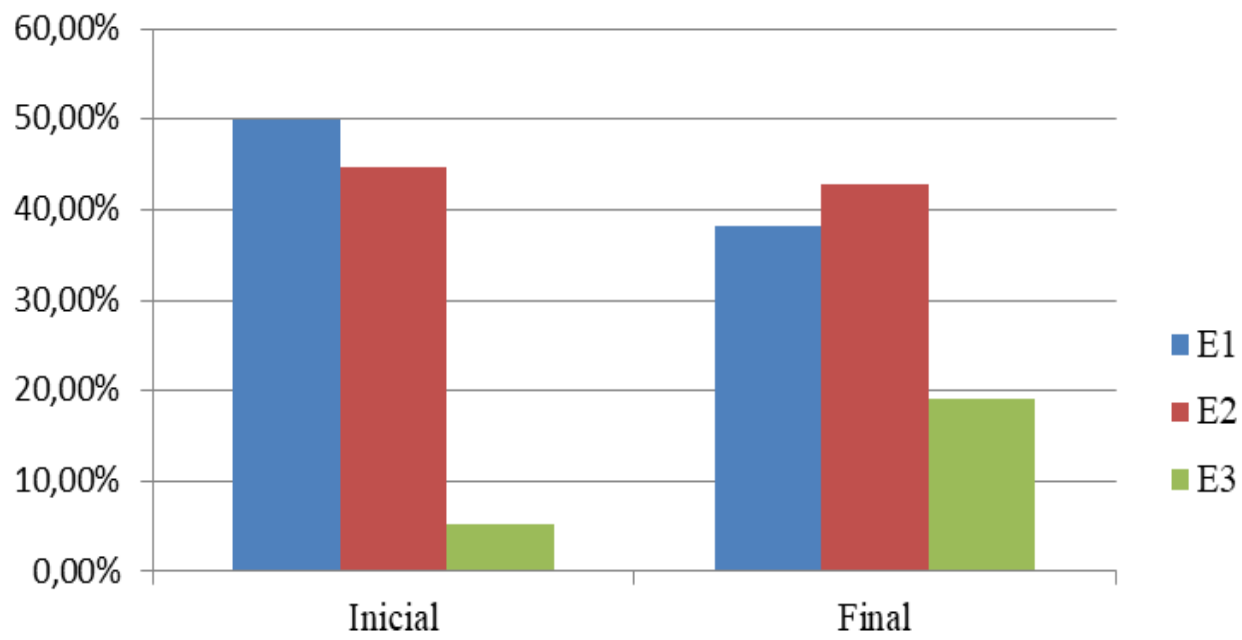

Fuente: Elaboración propia

En el gráfico se puede observar que, en las entrevistas iniciales, la mitad de las respuestas del alumnado (50\%) atribuye exclusivamente esta función al contexto familiar (nivel o valor 1), posición que disminuye en las entrevistas finales, con un $38 \%$. Respecto al segundo nivel, que en esta categoría está representado por una valoración genérica del papel de la educación formal, puede apreciarse que tanto en las entrevistas iniciales como en las finales hay un porcentaje de respuestas muy similar, aunque disminuye levemente en las finales (43\% frente a un $45 \%$ ). En el tercer nivel (valor 3 ) se han incluido valoraciones donde se afirmaba el relevante papel que ejerce la educación formal en la educación para la ciudadanía en interacción con las influencias del contexto social (especialmente el familiar); en las entrevistas iniciales las unidades de información en este nivel resulten escasas (5\%), sin embargo, las entrevistas que se llevaron a cabo tras finalizar la asignatura muestran un claro aumento de aportaciones (19\%).

Como unidades de información ejemplificadoras: 
Valor 1: "Yo pienso eso también, que en la familia es donde se empieza a educar en valores, que es importante como ciudadano, que es importante tener unos valores. Entonces yo pienso que en la familia es donde se inicia, donde empiezan a educarnos de esa manera, pero también al tú integrarte, al incluirte, digamos, en la sociedad externa, en el colegio, en la calle con los amigos... también vas comparando y vas también conociendo otros valores de otras personas." [Alumna MA; Grupo 3, entrevista inicial].

Valor 2: "Yo sobre todo en mi casa y en mi colegio. Por ejemplo, cada año ponían dos valores como fundamentales y lo iban recordando. Se hacían actividades y cada año tratábamos dos diferentes, como la tolerancia y la solidaridad. Entonces claro, pues no sólo que se pongan esas palabras y digas 'esto', no, pero se muestra, vemos vídeos, vemos cosas. Que no solo se quede en el aire, sino que realmente se trabaje y veamos cómo podemos tener esos valores." [Alumna MART; Grupo 3, entrevista final].

Categoría A: Aportación de la asignatura "Ciudad y ciudadanía en la Educación Infantil" a la construcción del modelo de ciudadanía

La última categoría se refiere a cómo la asignatura "Ciudad y ciudadanía en la Educación Infantil” ha influido en el proceso de construcción del modelo de ciudadanía de los y las estudiantes.

GRÁFICO 5. Categoría A: Aportación de la asignatura "Ciudad y ciudadanía en la Educación Infantil" a la construcción del modelo de ciudadanía

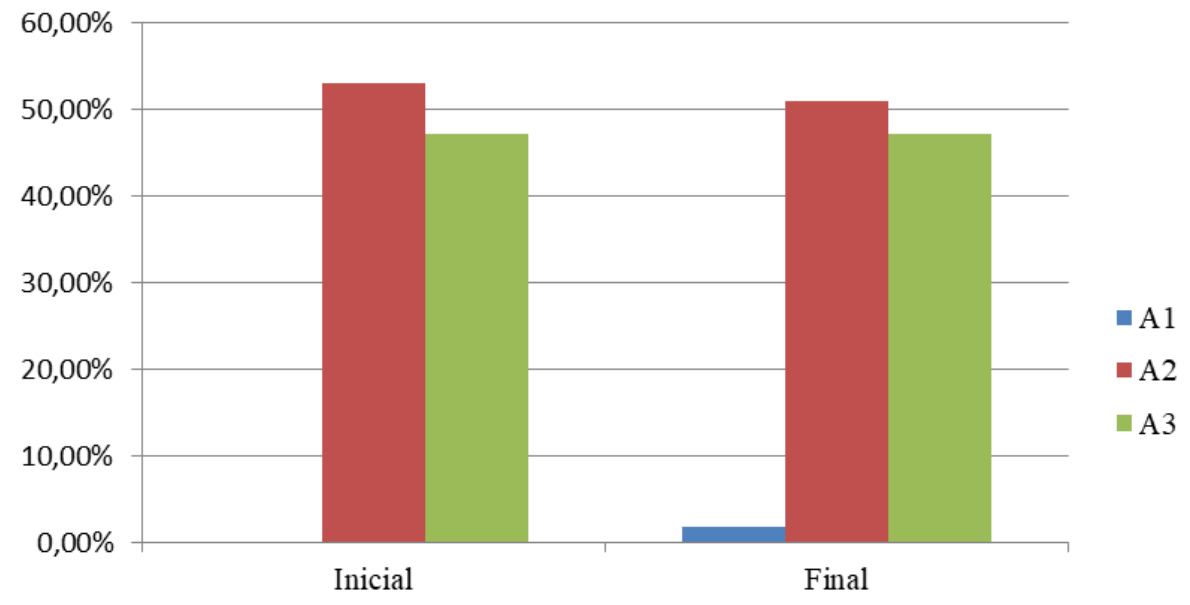

Fuente: Elaboración propia

Como puede verse en el gráfico 5, aparece un primer nivel (valor 1) con las respuestas en las que no se menciona la aportación de la asignatura en dicho proceso. En las primeras entrevistas, existe un $0 \%$ de unidades, algo que aumenta solo al $2 \%$ en las finales. Respecto al nivel 2 , se recogen respuestas que mencionan de manera puntual aportaciones, recursos metodológicos y comportamientos del docente en la asignatura. De este modo, resulta destacable cómo, incluso en las entrevistas iniciales (realizadas en las primeras semanas de desarrollo de la asignatura) ya el alumnado percibía una relación directa entre la asignatura y su construcción personal del modelo de ciudadanía (53\% de unidades), lo que se mantiene de manera similar en las finales (51\%). Para finalizar, el último nivel presenta una situación estable, pues tanto en las entrevistas iniciales como en las finales se reconoce que el modelo didáctico empleado en la asignatura aporta un modelo global de ciudadanía, con un $47 \%$ de unidades de información en ambos momentos.

A modo de ejemplos, estas unidades de información: 
Valor 2: "Yo creo que algo que nos marcó muchísimo fue la película, la de "Home" (actividad de la asignatura basada en el análisis de este documental), que te hace como un paso por todos los problemas que hay ahora mismo sociales en el mundo, sobre todo de medio ambiente ¿no? Que es verdad que ahí sí nos planteamos un montón de cosas de las que no somos conscientes, yo creo que de esa película se acuerda todo el mundo y a bastante gente como que le llamó la atención." [Alumna B; Grupo 1, entrevista final].

Valor 3: "Que no es el mismo método de 'yo te enseño una cosa y después, el examen'. Porque eso realmente te lo estudias, haces el examen y luego se te olvida. Yo creo que es mucho mejor trabajar con ellos, leernos textos, a lo mejor analizando un poco... Y después ya, a partir de todo eso, hicimos nuestra práctica final. Que yo lo veo mucho más constructivo para que realmente se nos quede. Porque el estudiar un temario es como todas las asignaturas; entonces es como cambiar un poco la metodología." [Alumna MART; Grupo 3, entrevista final].

\section{DISCUSIÓN Y CONCLUSIONES}

Tomando como referencia los resultados en ambas macrocategorías, podemos aproximar respuestas a los problemas planteados en la investigación acerca del proceso de construcción de la ciudadanía en el alumnado universitario que se prepara para la profesión docente (en Educación Infantil).

Como síntesis de la primera macrocategoría, "Concepción de ciudadanía", cabe destacar que el alumnado, a lo largo del desarrollo de la asignatura, ha ido abandonando la idea de la ciudadanía como un concepto superficial centrado en rasgos externos ligados meramente a derechos, normas y deberes. Desde esa concepción predominante poco a poco van evolucionando hacia una noción de ciudadanía basada en un mayor grado de compromiso con los problemas socioambientales, un modelo que hemos postulado en el marco teórico (Bolívar, 2016; Guérin, Ploeg, \& Sins, 2013; Tonge, Mycock, \& Jeffery, 2012; Wintersteiner et al., 2015).

Esta evolución va de la mano de la conexión que percibe el alumnado entre los propios actos y dichos problemas, considerando que sus actos pueden conllevar determinadas consecuencias, si bien esto parece más fruto de una buena voluntad que de una comprensión coherente, pues pocas veces asumen las consecuencias de sus actos como ciudadanos, y acaban atribuyendo la responsabilidad a agentes externos. Ello puede deberse a determinadas barreras y obstáculos de aprendizaje (De Alba Fernández y García Pérez, 2017; García Pérez, 2018), consolidados en el contexto del modelo social dominante, en el que apenas tiene presencia la idea de responsabilidad ciudadana. De hecho, aparecen escasas experiencias de participación real, de liderazgo y de toma de iniciativa hacia la movilización social y la participación ciudadana.

En la segunda macrocategoría, “Aprendizaje y evolución de la concepción de ciudadanía”, se analiza cómo ha cambiado dicha concepción en el alumnado a partir de su interacción en diferentes contextos sociales y formativos. En ese aspecto se da cierto equilibrio entre la influencia de los grupos sociales más cercanos, como la familia, y de la educación formal, con una interacción constante entre ambos; sin embargo, a partir de la experiencia en la asignatura cursada se percibe claramente la influencia del contexto educativo universitario en la concepción de ciudadanía. Tomando como referencia la programación del docente, consideramos que las actividades de la asignatura, así como el modelo metodológico aplicado en el aula, son factores influyentes, pues se han generado situaciones de enseñanza y aprendizaje que han acercado al alumnado a los problemas sociales y ambientales reales y a un papel activo y crítico en los procesos de participación.

Ya desde el inicio de la asignatura, se aprecia en el alumnado un impacto positivo en la construcción del propio modelo de ciudadanía. En las entrevistas se ha destacado la incidencia de determinadas actividades en su concienciación sobre los problemas socioambientales, como el visionado de películas documentales o el análisis de informes sobre la situación mundial. Asimismo, han sido importantes las reflexiones sobre las posibilidades que ofrece el propio currículum oficial 
para educar en ciudadanía y, sobre todo, el conocimiento de proyectos de educación de los niños y niñas para la participación ciudadana. La culminación de la asignatura con el diseño, por parte de los grupos de trabajo, de un proyecto o propuesta de intervención didáctica contribuyó a consolidar el vínculo con la realidad de la enseñanza.

Por lo demás, resulta relevante que los alumnos y alumnas fueron capaces de percibir el papel del docente como una referencia para construir un modelo de ciudadanía deseable. En ese sentido, hay referencias explícitas a cómo se ha percibido la aplicación real en las clases del modelo didáctico que la asignatura pretendía transmitir, confirmándose que el aprendizaje realmente significativo para llegar a ser un buen docente se desarrolla viviendo dicho modelo en la experiencia formativa cotidiana, sin olvidar que ello habría de complementarse con experiencias personales de participación ciudadana habidas en contextos sociales reales, como se destaca en diversas investigaciones (Daly, Schugurensky, \& Lopes, 2009; Novella, Agud, Llena y Trilla, 2013; Schugurensky \& Myers, 2003).

En definitiva, la utilización en el aula de una metodología basada en el tratamiento de problemas reales, en el aprendizaje significativo, en la horizontalidad y en la construcción del conocimiento, ha permitido al alumnado sentirse escuchado y valorado. Ello ha configurado un contexto formal de aprendizaje que promueve la construcción de una ciudadanía con una visión más crítica y con herramientas útiles para tomar decisiones fundamentadas y emprender cambios a nivel personal y colectivo, y a escala local y global. Estos rasgos del modelo formativo también han sido destacados en diversas investigaciones a las que hemos hecho referencia anteriormente (Estepa, 2012; García Pérez, De Alba Fernández y Navarro Medina, 2015; González Valencia y Sant, 2014; Willemse et al., 2015).

La validación de este modelo formativo, junto a la evolución de la concepción de ciudadanía hacia formulaciones más complejas, son, en síntesis, las aportaciones de la investigación presentada que consideramos más relevantes. Corresponde, en todo caso, a las instituciones encargadas de la formación del profesorado, y específicamente, en el marco universitario, a las Facultades de Educación, replantearse en profundidad la formación de dicho profesorado, como los resultados de diversas investigaciones, asimismo, postulan (Estellés y Romero, 2018; Krichesky et al., 2011; Molina, Miralles y Ortuño, 2013).

\section{Referencias}

Álvarez-García, O., Sureda-Negre, J. y Comas-Forgas, R. (2018). Evaluación de las competencias ambientales del profesorado de Primaria en formación inicial: estudio de caso. Enseñanza de las Ciencias: Revista de Investigación y Experiencias Didácticas, 36(1), 117-141. DOI: 10.5565/rev/ensciencias. 2338

Bardin, L. (1991). Análisis de contenido. Madrid: Akal.

Bisquerra, R. (coord.) (2009). Metodología de la investigación educativa. Madrid: La Muralla, $2^{\mathrm{a}}$ ed.

Bolívar, A. (2016). Educar democráticamente para una ciudadanía activa. Revista Internacional de Educación para la Justicia Social, 5(1), 69-87. DOI: 10.15366/riejs2016.5.1

Bourn, D., Huntan, F., \& Bamber, Ph. (2017). A review of education for sustainable development and global citizenship education in teacher education. París: UNESCO. Recuperado de https://unesdoc.unesco.org/ark:/48223/pf0000259566

Buendía, L., Colás, P. y Hernández, F. (2012). Métodos de investigación en psicopedagogía. Madrid: Mcgraw-Hill / Interamericana de España, S.A.U.

Comisión Europea (2005). La educación para la ciudadanía en el contexto escolar europeo. Bruselas: Eurydice. Recuperado de https://www.oei.es/historico/valores2/055ES.pdf

Daly, K., Schugurensky, D., \& Lopes, K. (ed.) (2009). Learning Democracy by Doing: Alternative Practices in Citizenship Education and Participatory Democracy. Toronto: Transformative Learning Centre, OISE/UT. 
De Alba Fernández, N. y García Pérez, F.F. (2017). L’implication des professeurs dans les programmes d'éducation à la participation citoyenne. Le cas du «Parlement des Jeunes » à Séville. In Ph. Haeberli, M. Pagoni et O. Maulini (dirs.), La participation des élèves: effet de mode ou nécessité? (pp. 219-236). Paris: L’Harmattan.

De Manuel Jerez, E. y Donadei, M. (2018). La extensión universitaria como dinamizadora de la función social compleja de la universidad. Estoa, Revista de la Facultad de Arquitectura y Urbanismo de la Universidad de Cuenca, 7(14), 115-127. DOI: 10.18537/est.v007.n014.a09

Estellés, M. \& Romero, J. (2018). Tacit assumptions of citizenship education: A case study in Spanish initial teacher education. Education. Citizenship and Social Justice. First Published May 21, 2018. DOI: $10.1177 / 1746197918771336$

Estepa, J. (2012). La formación del profesorado para enseñar la participación en el Grado de Maestro y en el Máster en Profesorado de Secundaria. Una alternativa basada en los problemas prácticos profesionales. En N. De Alba, F. F. García Pérez y A. Santisteban (coords.), Educar para la participación ciudadana en la enseñanza de las Ciencias Sociales (vol. 2, pp. 211-220). Sevilla: Díada. Recuperado de http://www.didactica-cienciassociales.org/publicaciones_archivos/2012-sevilla-XXIII-Simposio-DCS_II.pdf

García Pérez, F.F. (2006). Formación del profesorado y realidades educativas: una perspectiva centrada en los problemas prácticos profesionales. En J. M. Escudero y A. Luis (Eds.), La formación del profesorado y la mejora de la educación. Políticas y prácticas (pp. 269-310). Barcelona: Octaedro.

García Pérez, F.F. (2018). Are Teachers Prepared to Educate in Citizenship? Some Conclusions from Research in Andalusia, Spain. In J. A. Pineda-Alfonso, N. De Alba-Fernández, \& E. NavarroMedina, Handbook of Research on Education for Participative Citizenship and Global Prosperity (pp. 409-430). Hershey PA, USA: IGI Global.

García Pérez, F.F., De Alba Fernández, N. y Navarro Medina, E. (2015). La formación inicial del profesorado para enseñar ciudadanía. Experiencias en los niveles de grado y de máster. En B. Borghi, F. F. García Pérez y O. Moreno Fernández (eds.), Novi Cives. Cittadini dall’infanzia in poi (pp. 137-148). Bologna: Pàtron Editore.

García Pérez, F.F., Moreno Fernández, O. y Rodríguez Marín, F. (2015). Problemas del mundo y educación: hacia una ciudadanía planetaria. En B. Borghi, F. F. García Pérez y O. Moreno Fernández (eds.), Novi Cives. Cittadini dall'infanzia in poi (pp. 33-41). Bologna: Pàtron Editore.

García Pérez, F.F. y Porlán, R. (2000). El Proyecto IRES (Investigación y Renovación Escolar). Biblio 3W. Revista Bibliográfica de Geografía y Ciencias Sociales, V(205), 16 de febrero de 2000. Recuperado de http://www.ub.edu/geocrit/b3w-205.htm

García Pérez, F.F. y Porlán, R. (2017). Los principios didácticos y el modelo didáctico personal. En R. Porlán (coord.), Enseñanza universitaria. Cómo mejorarla (pp. 93-104). Madrid: Morata.

Gómez-Rodríguez, A.E. y García-Ruiz, C.R. (2019). Educación para la Ciudadanía en España. Una asignatura para la confrontación ideológica y política. Didáctica de las ciencias experimentales y sociales, 37, 51-66. DOI: 10.7203/DCES.37.14318

González Valencia, G. y Sant, E. (2014). ¿Qué debe saber el profesorado para educar a ciudadanos críticos y participativos? En J. Pagès y A. Santisteban (eds.), Una mirada al pasado y un proyecto de futuro: investigación e innovación en didáctica de las ciencias sociales (vol. 2, pp. 295-303). Barcelona: Universitat Autònoma de Barcelona, Servei de Publicacions, y Asociación Universitaria de Profesorado de Didáctica de las Ciencias Sociales. Recuperado de http://didactica-ciencias-sociales.org/wp-content/uploads/2013/07/XXVSIMPO2.pdf

Guérin, L.J.F., Ploeg, P. A. van der, \& Sins, P. H. M. (2013). Citizenship education: the feasibility of a participative approach. Educational Research, 55(4), 427-440. DOI: 10.1080/00131881.2013.844945 
Krichesky, G. J., Martínez-Garrido, C., Martínez Peiret, A.M., García Barrera, A., Castro Zapata, A. y González Bustamante, A. (2011). Hacia un programa de formación docente para la justicia social. REICE. Revista Iberoamericana sobre Calidad, Eficacia y Cambio en Educación, 9(4), 64-77. Recuperado de http://www.rinace.net/reice/numeros/arts/vol9num4/art3.pdf

Krippendorff, K. (1990). Metodología de análisis de contenido. Teoría y práctica. Barcelona: Paidós. Manzano-Arrondo, V. (2011). La universidad comprometida. Barcelona: Hipatia.

Martínez-Usarralde, M.J., Lloret-Catalá, C. y Mas-Gil, S. (2017). Responsabilidad Social Universitaria (RSU): Principios para una universidad sostenible, cooperativa y democrática desde el diagnóstico participativo de su alumnado. Archivos Analíticos de Políticas Educativas, 25(75). DOI: 10.14507/epaa.25.2769

Molina, S., Miralles, P. y Ortuño, J. (2013). Concepciones de los futuros maestros de Educación Primaria sobre formación cívica y ciudadana. Educatio Siglo XXI, 31(1), 105-126. Recuperado de https://revistas.um.es/educatio/article/view/175361/148441

Naciones Unidas (2015). Objetivos de Desarrollo del Milenio. Informe de 2015. Nueva York: Naciones Unidas. Recuperado de https://www.un.org/es/millenniumgoals/pdf/2015/mdgreport-2015_spanish.pdf

Novella, A.M., Agud, I., Llena, A. y Trilla, J. (2013). El concepto de ciudadanía construido por jóvenes que vivieron experiencias de participación infantil. Bordón. Revista de Pedagogía, 65(3), 93-108. Recuperado de https://recyt.fecyt.es/index.php/BORDON/article/view/23166

Observatorio de la Cooperación Universitaria al Desarrollo (2016). Resumen de los Diálogos sobre Universidad y Desarrollo Sostenible. AECID, 10 de marzo de 2016. Recuperado de http://www.ocud.es/es/pl59/actividades-propias-y-en-red/jornadas-ocud/id2086/resumen-delos-dialogos-sobre-universidad-y-desarrollo-sostenible.htm

Pagès, J. y Santisteban, A. (coords.) (2011). Les qüestions socialment vives i l'ensenyament de les ciències Socials. Barcelona: Servei de Publicacions de la Universitat Autònoma de Barcelona. Col. Documents, 9.

Romero Morante, J. (2012). ¿Socialización política "programada"? Una aproximación dilemática a la investigación sobre las complejas relaciones entre educación y participación ciudadana. En N. de Alba, F.F. García y A. Santisteban (eds.), Educar para la participación ciudadana en la enseñanza de las Ciencias Sociales, (vol. I, pp. 257-275). Sevilla: Díada.

Romero Morante, J. (2018). Lo que no suele discutirse en el debate sobre las Humanidades y la educación democrática. En B. Tosar, A. Santisteban y J. Pagès (eds.), Què està passant al món? Què estem ensenyant? Per un ensenyament de les ciències socials centrat en els problemes, la justícia social i la ciutadania global (pp. 33-54). Barcelona: GREDICS (Grup de Recerca en Didàctica de les Ciències Socials), Universitat Autònoma de Barcelona.

Schugurensky, D. y Myers, J. (2003). Learning to Teach Citizenship: A Lifelong Learning Approach. Encounters on Education, 4, 145-166. DOI: 10.24908/eoe-ese-rse.v4i0.663

Schulz, W., Ainley, J., Fraillon, J., Losito, B., Agrusti, G. y Friedman, T. (2017). Becoming Citizens in a Changing World. IEA International Civic and Citizenship Education Study 2016. International Report. Amsterdam: IEA/Springer Open. Recuperado de https://www.uvm.dk//media/filer/uvm/int/180301-iccs-2016-international-report.pdf

Tonge, J., Mycock, A., \& Jeffery, B. (2012). Does citizenship education make young people betterengaged citizens? Political Studies, 60(3), 578-602. DOI: 10.1111/j.1467-9248.2011.00931.x

UNESCO (1998). Declaración mundial sobre la educación superior en el siglo XXI: visión y acción. París:

UNESCO.

Recuperado

de

http://www.unesco.org/education/educprog/wche/declaration_spa.htm

UNESCO (2009). Conferencia Mundial sobre la Educación Superior-2009: La nueva dinámica de la educación superior y la investigación para el cambio social y el desarrollo. Comunicado (8 de julio de 2009). París: UNESCO. Recuperado de http://www.unesco.org/education/WCHE2009/comunicado_es.pdf 
UNESCO (2014). Shaping the Futur We Want: UN Decade of Education for Sustainable Development (2005-2014). Final Report. París: UNESCO. Recuperado de https://unesdoc.unesco.org/ark:/48223/pf0000230171

Willemse, T.M., Ten Dam, G., Geijsel, F., Van Wessum, L., \& Volman, M. (2015). Fostering teachers' professional development for citizenship education. Teaching and Teacher Education, 49, 118-127. DOI: 10.1016/j.tate.2015.03.008

Wintersteiner, W., Grobbauer, H., Diendorfer, G. y Reitmair-Juárez, S. (2015). Global Citizenship Education: Citizenship Education for Globalizing Societies. Vienna: Zentrum für Friedensforschung und Friedenspädagogik. Recuperado de http://www.demokratiezentrum.org/fileadmin/media/pdf/Materialien/GlobalCitizenshipEduca tion_Final_english.pdf

\section{CÓMO CITAR ESTE ARTÍ́CULO}

García Pérez, F.F., Burgos Sánchez, M. y Guerrero Fernández, A. (2020). La construcción de la ciudadanía en la formación inicial del profesorado de Educación Infantil. Didáctica de las ciencias experimentales y sociales, 39, 47-64. DOI: 10.7203/DCES.39.17121 
